\title{
Cough Augmentation in Subjects With Duchenne Muscular Dystrophy: Comparison of Air Stacking via a Resuscitator Bag Versus Mechanical Ventilation
}

\author{
Michel Toussaint PhD, Kurt Pernet MPhysio, Marc Steens MPhysio, Jurn Haan MD, and \\ Nicole Sheers MPhysio
}

\begin{abstract}
BACKGROUND: Air stacking improves cough effectiveness in people with Duchenne muscular dystrophy (DMD) and respiratory muscle weakness. However, it is not known whether air stacking is more effective via a resuscitator bag or a home ventilator. METHODS: This prospective randomized study investigated the effect of air stacking via a volume-cycled home ventilator versus via a resuscitator bag in participants with DMD. Maximum insufflation capacity and peak expiratory flow during spontaneous (cough peak flow) and air stacking-assisted cough maneuvers (air stacking-assisted cough peak flow) were measured. RESULTS: Fifty-two adult DMD subjects receiving noninvasive ventilation were included in the study: 27 participants performed air stacking via their home ventilator (home-ventilator group; age $=25.3 \pm 5.1 \mathrm{y}$; forced vital capacity $(\mathrm{FVC})=$ $809 \pm 555 \mathrm{~mL}$ ), and 25 participants used a resuscitator bag (resuscitator-bag group; age $=24.7 \pm 5.7 \mathrm{y}$, FVC $=807 \pm 495 \mathrm{~mL}$ ). Following a single training session, air stacking could be performed successfully by $89 \%$ (home ventilator) and $88 \%$ (resuscitator bag) of participants. There were comparable maximum insufflation capacities $(1,481 \mathrm{~mL}$ for the home-ventilator group vs $1,344 \mathrm{~mL}$ for the resuscitator-bag group, $P=.33$ ) and mean air stacking-assisted cough peak flow values $(199 \mathrm{~L} / \mathrm{min}$ for the home-ventilator group vs $186 \mathrm{~L} / \mathrm{min}$ for the resuscitator-bag group, $P=.33$ ) between techniques. Air stacking-assisted cough peak flow increased significantly compared with baseline in both groups (mean increase: $+51 \%$ [home ventilator] vs $+49 \%$ [resuscitator bag], $P<.001$ ), with individual air stacking-assisted cough peak flow improvements ranging from -20 to $245 \%$. CONCLUSIONS: Cough augmentation is an important component of the respiratory management of people with a neuromuscular disorder. No difference in cough effectiveness as measured by air stacking-assisted cough peak flow was found in air stacking via a ventilator compared with via a resuscitator bag. Both methods achieved mean air stacking-assisted cough peak flow values of $>160 \mathrm{~L} / \mathrm{min}$. Provision of an inexpensive resuscitator bag can effectively improve cough capacity, and it is simple to use, which may improve access to respiratory care in people with DMD. Key words: airway clearance; air stacking; chest physiotherapy; cough; Duchenne muscular dystrophy. [Respir Care 2016;61(1):61-67. ( 2016 Daedalus Enterprises]
\end{abstract}

Introduction

Duchenne muscular dystrophy (DMD) is a rapidly progressive neuromuscular disorder, affecting 1 in 3,300 male

Dr Toussaint, Mr Pernet, Mr Steens, and Dr Haan are affiliated with Ziekenhuis Inkendaal, Vlezenbeek, Belgium. Ms Sheers is affiliated with the Victorian Respiratory Support Service and Institute for Breathing and Sleep, Austin Health, Heidelberg, Victoria, Australia. births. ${ }^{1}$ Skeletal, respiratory, and cardiac muscle weakness develop as age increases, leading to loss of ambulation, chest wall deformity, chronic ventilatory failure, and

\footnotetext{
Correspondence: Michel Toussaint $\mathrm{PhD}$, Rehabilitation Hospital, Inkendaal, Inkendaalstraat 1, B-1602 Vlezenbeek (Brussels), Belgium. E-mail: michel.toussaint@inkendaal.be.
}

DOI: $10.4187 /$ respcare.04033 


\section{Cough Augmentation for Duchenne Muscular Dystrophy}

cardiomyopathy. Without ventilatory support, death due to respiratory failure occurs at around $20 \mathrm{y}$ of age. ${ }^{2,3}$ Noninvasive mechanical ventilation (NIV) has been demonstrated to slow the decline in respiratory function and improve median survival to $30-35 \mathrm{y}^{4,5}$; however, ventilation is just one aspect of patient respiratory care. Recurrent atelectasis, inability to clear secretions, and respiratory infections also increase morbidity and mortality. Respiratory tract infections are the commonest cause of hospital admissions in people with DMD, ${ }^{6}$ and an ineffective cough as measured by cough peak flow has been associated with higher risks of pneumonia ${ }^{6}$ and need for tracheostomy. ${ }^{7}$

Effective airway clearance is thus an integral component of respiratory management. Retrospective cohort data suggest that survival improves when subjects, families, and home-care providers are taught effective cough assistance techniques 8,9 or when a care plan incorporating cough augmentation is implemented in the home. A number of techniques have been reported that augment inspiration, expiration, or both components of a cough. These include mechanical insufflation-exsufflation (in-exsufflation), manually assisted coughing via an abdominal thrust, and air stacking. Although the use of mechanical in-exsufflation has been advocated to maintain cough peak flow above proposed critical values of $160-270 \mathrm{~L} / \mathrm{min},{ }^{6,7}$ it requires expensive equipment (approximately $€ 5,000$ in Europe) and therefore is not widely accessible in countries with limited health-care resources. Even in countries with wellstructured healthcare systems, mechanical insufflation-exsufflation may not always be available in the community. Other cough augmentation techniques may improve cough effectiveness sufficiently but cost significantly less and thus be more accessible. Manually assisted coughing was found to benefit subjects whose forced vital capacity (FVC) was within $1,030-1,910 \mathrm{~mL}$, but the use of air stacking alone or in combination with manually assisted coughing produced effective cough peak flow values in subjects with FVC of $>340-560 \mathrm{~mL}$, suggesting that it is beneficial even for moderate to severely weak patients. ${ }^{10}$

\section{What Is Air Stacking?}

Air stacking involves taking multiple successive insufflations, breathing in without breathing out, to achieve lung maximum insufflation capacity before exhalation or coughing. When hyperinflated in this manner to maximum insufflation capacity, subsequent cough maneuvers are performed with increased inspiratory volume. Air is passively delivered using a positive pressure device, such as a ventilator or resuscitator bag, ${ }^{11}$ or via glossopharyngeal breathing. ${ }^{12}$ When using glossopharyngeal breathing or a ventilator, patients need to coordinate both opening of their glottis during each insufflation and closure at the end in order to hold the stacked air and increase inspiratory capacity. For people with impaired

\section{QUICK LOOK}

\section{Current knowledge}

Cough augmentation is an important component of the respiratory management of patients with neuromuscular disorders. Insufflation and exsufflation promote lung expansion, improve oxygenation, and enhance secretion removal. Augmented cough can be accomplished with a manual resuscitator, a mechanical ventilator, or cough assist device.

\section{What this paper contributes to our knowledge}

In a group of subjects with Duchenne muscular dystrophy, the use of a volume-cycled ventilator or a manual resuscitator to stack breaths resulted in similar cough peak flows. Both methods achieved cough peak flow $>160$ $\mathrm{L} / \mathrm{min}$. The use of a manual resuscitator and breath stacking for augmented coughing can be successful in resource constrained environments.

glottic function, a one-way valve can be placed in the circuit of a resuscitator bag to enable air stacking. The number of successive insufflations required to achieve one maximum insufflation capacity-stacked breath varies according to the individual and the air-stacking technique used.

Clinically, air stacking can be used during periods of acute respiratory infection or prophylactically as part of daily respiratory management. A retrospective cohort study in DMD suggested that the rate of decline in lung function was slowed after twice daily air stacking was initiated..$^{13}$ It is simple to teach and trial, and generally patients and their caregiver(s) are able to learn this during a single training session. Performed as a cough augmentation technique, air stacking significantly improves short-term cough effectiveness with dramatic gains in cough peak flow above proposed critical values. ${ }^{10,14-17}$

Despite the clinical use of air stacking, there is no evidence comparing available techniques. Although air stacking can be performed independently or with minimal assistance via a home ventilator, models that allow this are expensive and may not be accessible to all. By contrast, a resuscitator bag is inexpensive (between $€ 20$ and $€ 50$ ) and universally available. The disadvantage of this technique is that it typically requires a second person to hold a full face mask on the patient's face and to insufflate the lungs by compressing the bag (Fig. 1). Given that both techniques have been described in the literature with no comparative data to date, the aim of this study was to compare the effectiveness of air stacking using a resuscitator bag versus air stacking using a home ventilator on cough augmentation. 


\section{Cough Augmentation for Duchenne Muscular Dystrophy}

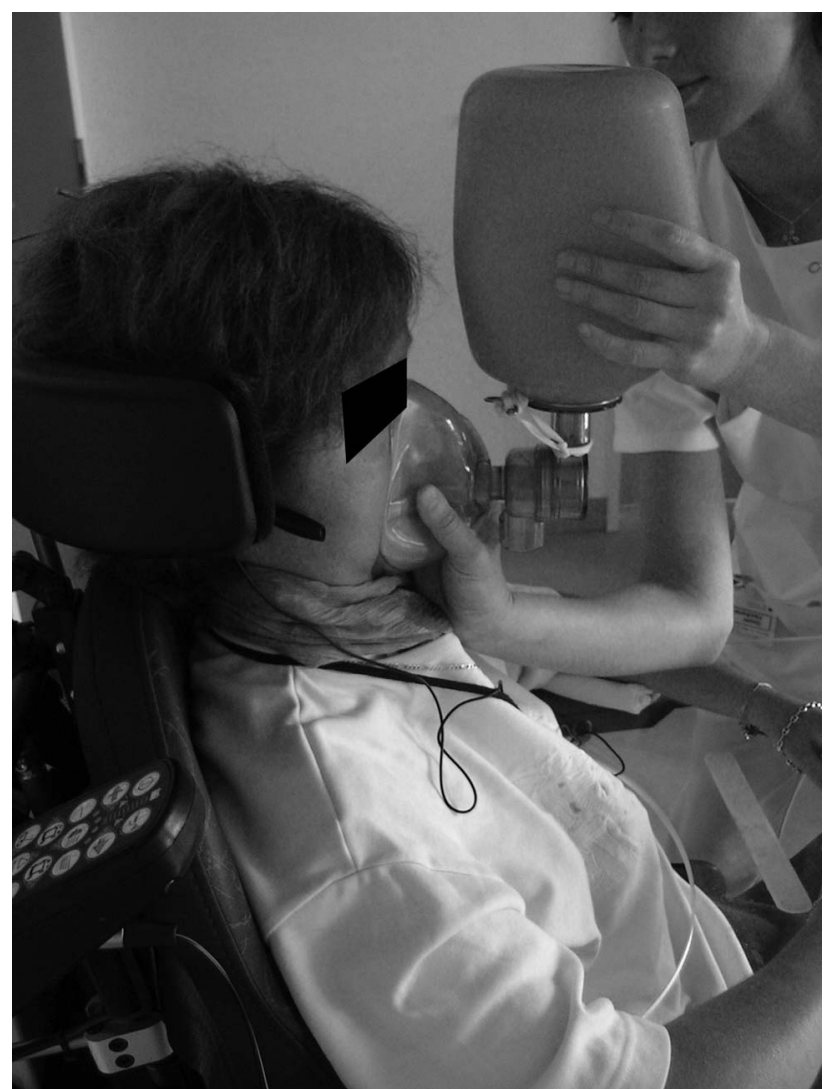

Fig. 1. Air stacking via a resuscitator bag using a full face mask.

\section{Methods}

\section{Study Design and Participants}

A prospective comparative parallel group study was performed. Participants were allocated by coin toss to a single session of either air stacking via home ventilator or air stacking via a resuscitator bag at a routine clinical visit.

All adult patients ( $\geq 18 \mathrm{y}$ ) with DMD and requiring NIV followed up at the Neuromuscular Excellency Centre and Centre for Home Mechanical Ventilation, Inkendaal Rehabilitation Hospital in a 2-y period (from January 2012 to December 2013) were considered for study inclusion. They all received volume cycle ventilation. Exclusion criteria included inability to perform lung function tests, tracheostomy in situ, prior formal training in air stacking, and respiratory instability defined as acute respiratory failure. NIV was instituted according to usual clinical practice at our Centre. ${ }^{18,19}$ Routine reviews were then scheduled every 6 months, alternating between an in-patient or out-patient visit. Informed consent was obtained before study inclusion. This study was approved by the Ethics Committee at our institution.

\section{Outcome Measures}

The primary outcome measure was air stacking-assisted cough peak flow. Participants were instructed to perform the randomly allocated air stacking technique (home ventilator or resuscitator bag) to maximal insufflation and then cough into a mask, in the sitting position, without additional manual assistance or abdominal compression. Lung volume was recorded from a maximal effort unassisted breath (FVC) and following an air stacking-assisted breath (maximum insufflation capacity). Maximal expiratory pressure $\left(\mathrm{P}_{\mathrm{Emax}}\right)$ was recorded from total lung capacity as per American Thoracic Society/European Respiratory Society guidelines. ${ }^{20}$ Measures of cough effectiveness and respiratory function were assessed via a heated Fleisch No. 2 pneumotachometer (Metabo, Lausanne, Switzerland) in the seated position, with the best of 3 trials recorded. FVC testing was performed according to American Thoracic Society/European Respiratory Society reference values. ${ }^{21}$ The testing procedure was designed according to the methodology described in a previous study by our group. ${ }^{18}$

\section{Study Procedure}

Following completion of routine clinical measures (baseline assessment), including spirometry and respiratory muscle strength (FVC and $\mathrm{P}_{\text {Emax }}$ ), unassisted cough peak flow, manually assisted cough peak flow, and ventilation-free time, defined as the cumulative spontaneously breathing duration in a 24-h period, participants were offered a period of $10 \mathrm{~min}$ of rest, during which the procedure of air stacking was explained in detail. As reported previously, ${ }^{10}$ manually assisted cough peak flow was achieved after a maximum unassisted spontaneous breath followed by a thoracic-abdominal thrust done by the therapist. After this 10-min period of rest, subjects were taught and trained for $2 \mathrm{~min}$ in both air stacking techniques (home ventilator and resuscitator bag). The number of successive insufflations required for each participant to optimize their technique was assessed and individualized during this training. Unassisted cough peak flow and manually assisted cough peak flow were measured before air stacking as part of the baseline measures. Following training in both techniques and then randomization, participants rested for $5 \mathrm{~min}$. They were then asked to perform the allocated air stacking technique as per their individualized prescription and then perform a cough with forced expiration into a mask held by a therapist. The best air stacking-assisted cough peak flow and maximum insufflation capacity values out of 3 air stacking maneuvers were retained for further analysis.

Air stacking via a home ventilator was performed using a volume-cycled home mechanical ventilator and nasal mask interface, with parameters unchanged from those used for nocturnal NIV. Air stacking via a resuscitator bag 
was conducted by an experienced physiotherapist from our Unit, using a 2-L bag (Resutator 2000, Dräger, Lübeck, Germany) and full face mask (Fig. 1). Instructions were tailored to the participant but might be, for example, as follows. Let your lungs fill with air from the ventilator/bag. Once this insufflation has finished, hold your breath and don't breathe out. Another insufflation will be delivered. Try to stack 2 or 3 of these successive insufflations. When your lungs feel like they're fully expanded, cough.

Following randomization, participants were asked to perform the allocated technique as per their individualized prescription and then perform a cough with forced expiration. The best air stacking-assisted cough peak flow value out of 3 air stacking maneuvers was retained for further analysis. Successful air stacking was defined as $>10 \%$ improvement in maximum insufflation capacity compared with a spontaneous FVC maneuver.

\section{Statistics}

A 2-way analysis of variance was used for group comparison. Data were computed in a database (Medcalc, Mariakerke, Belgium). Continuous variables are presented as mean \pm SD. Statistical significance was accepted at $P<.05$.

\section{Results}

Fifty-two subjects with DMD receiving NIV for a mean of $8.2 \pm 5.2$ y were included in this study. All participants were ventilated via volume-cycled ventilators and nasal mask nocturnally, with mean tidal volume $=720 \pm 90 \mathrm{~mL}$ and respiratory rate set at $22.2 \pm 4.2$ cycles $/ \mathrm{min}$. In addition, 35 subjects received diurnal mouthpiece intermittent positive-pressure ventilation for a mean of $4.5 \mathrm{y}$ as described previously. ${ }^{19}$ Participant characteristics are reported in Table 1.

Twenty-seven participants underwent air stacking via their home ventilator (home-ventilator group) and 25 via a resuscitator bag (resuscitator-bag group). Demographics, lung function values, and ventilation parameters were similar between the 2 groups. Air stacking could be performed by 89 and $88 \%$ of participants in the home-ventilator and resuscitator-bag groups, respectively (Table 2). The small number of subjects $(n=6 / 52)$ who could not increase their maximum insufflation capacity above their baseline FVC by $>10 \%$ did not allow a comparison in respiratory parameters between subjects who could perform the techniques and those who could not. There was no difference in air stacking-assisted cough peak flow between groups, and both techniques resulted in a significant mean increase compared with unassisted cough peak flow (home-ventilator group: $+67 \mathrm{~mL}, P<.001$; resuscitator-bag group: $+61 \mathrm{~mL}, P<.001)$. Similarly, there were comparable expired volumes between techniques, with maximum insuffla-
Table 1. Subject Characteristics

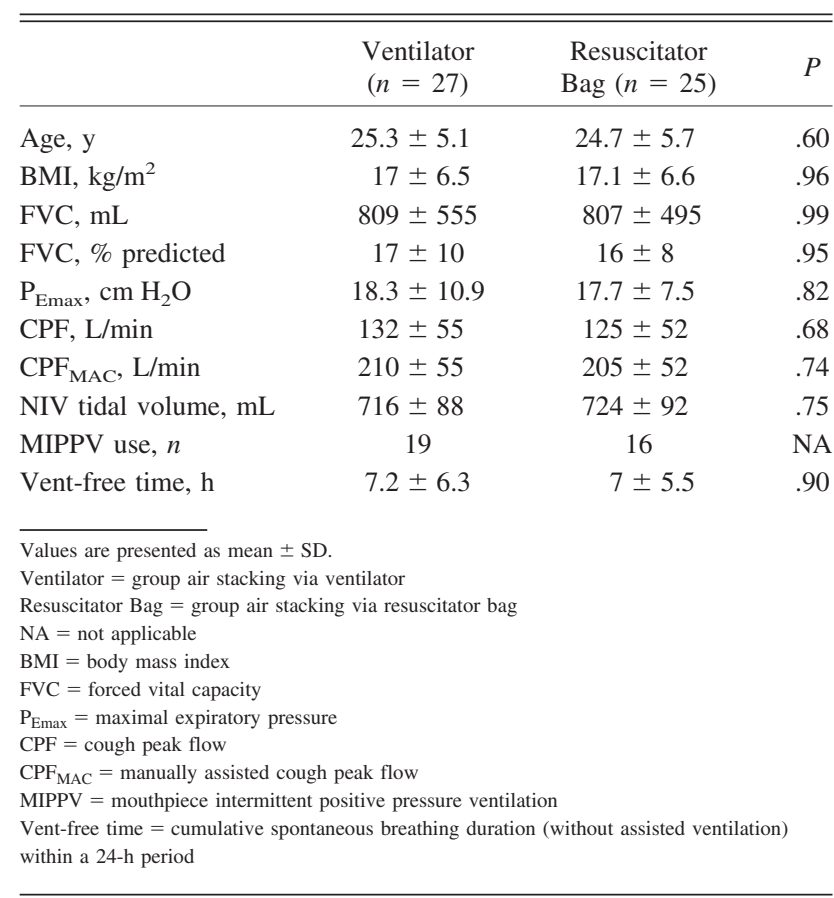

tion capacity values greater than spontaneous FVC in both groups (mean within group change: $+672 \mathrm{~mL}, P<.001$ for the home-ventilator group vs $+537 \mathrm{~mL}, P<.001$ for the resuscitator-bag group), and no difference in maximum insufflation capacity between groups (Table 2). Individual participant data depicting unassisted to air stacking-assisted change in cough peak flow and expired volume are presented in Figure 2. Individual cough peak flow change ranged from -20 to $245 \%$, with greater improvement in participants with lower baseline $\mathrm{P}_{\mathrm{Emax}}$ and FVC.

\section{Discussion}

Air stacking via a ventilator and air stacking via a resuscitator bag resulted in comparable short-term increases in cough peak flow in this prospective randomized trial comparing the 2 techniques in adults with DMD. According to a recent report, ${ }^{16}>80 \%$ of participants could successfully perform air stacking maneuvers regardless of the allocated technique, with a mean improvement in air stacking-assisted cough peak flow above spontaneous unassisted cough peak flow between $49 \%$ (resuscitator-bag group) and $51 \%$ (home-ventilator group). Importantly, both techniques achieved a mean cough peak flow value of $>160$ L/min, the proposed critical level necessary to avoid tracheostomy. ${ }^{7}$ However, a proportion of individuals using either the ventilator or the bag did not reach the threshold value of $160 \mathrm{~L} / \mathrm{min}$ (19\% vs $28 \%$, respectively, $P=.78$ ). The major reason for this failure was the low baseline 
Table 2. Comparison of Air Stacking via Ventilator Versus via Resuscitator Bag

\begin{tabular}{|c|c|c|c|}
\hline & Ventilator & Resuscitator Bag & $P$ \\
\hline Able to perform air stacking, $n(\%)$ & 24/27 (89) & $22 / 25(88)$ & NA \\
\hline Insufflations to maximal insufflation capacity, $n$ & $2.6 \pm 0.6$ & $1.8 \pm 0.6$ & $<.001$ \\
\hline $\mathrm{CPF}_{\mathrm{AS}}, \mathrm{L} / \mathrm{min}$ & $199 \pm 48$ & $186 \pm 50$ & .33 \\
\hline Maximal insufflation capacity, $\mathrm{mL}$ & $1,481 \pm 477$ & $1,344 \pm 520$ & .33 \\
\hline Expected expired volume, $\mathrm{mL}$ & $1,770 \pm 404$ & NA & NA \\
\hline Ventilator leak, $\mathrm{mL}$ & $289 \pm 468$ & NA & NA \\
\hline $\mathrm{P}_{\text {EmaxAS }}, \mathrm{cm} \mathrm{H} \mathrm{H}_{2} \mathrm{O}$ & $28 \pm 10$ & $26 \pm 9$ & .45 \\
\hline \multicolumn{4}{|c|}{$\begin{array}{l}\text { Values are presented as mean } \pm \mathrm{SD} \text {. or } n(\%) \text {. } \\
\mathrm{NA}=\text { not applicable } \\
\text { Insufflations to maximal insufflation capacity }=\text { successive insufflations before coughing } \\
\mathrm{CPF}_{\mathrm{AS}}=\text { air stacking-assisted cough peak flow } \\
\text { Maximal insufflation capacity }=\text { expired volume following an air stacking-assisted breath } \\
\text { Expected expired volume }=\text { expected volume if there were no leaks (number of successive insufflations } \times \text { tidal volume of the ventilator) } \\
\text { Ventilator leak }=\text { difference between expected expired volume and maximal insufflation capacity } \\
\mathrm{P}_{\text {EmaxAS }}=\text { maximal expiratory pressure following an air stacking-assisted breath }\end{array}$} \\
\hline
\end{tabular}

unassisted cough peak flow value. Our data suggest that subjects with unassisted cough peak flow $<90 \mathrm{~L} / \mathrm{min}$ were not able to reach an assisted cough peak flow of $>160$ L/min (Fig. 2, A and B) using air stacking alone and would therefore be ideal candidates to use a combination of air stacking and manually assisted coughing or mechanical in-exsufflation to augment cough. Future studies will be required to confirm this hypothesis.

The magnitude of improvement in cough peak flow with air stacking varies in the literature between 19 and 87\%,15,22 possibly due to different populations, baseline lung function, the specific air stacking technique used, and the expertise of the therapist. FVC, $\mathrm{P}_{\text {Emax }}$, and inspiratory capacity are correlated with unassisted cough peak flow value, ${ }^{23}$ with those subjects with poorer lung function recording smaller cough peak flow values but benefitting most from cough augmentation techniques. ${ }^{10,15}$ Interestingly, recent data suggest that the ideal assisted insufflation before assisting cough should be submaximal rather than maximal, even in those patients with severely reduced compliance of the respiratory system. ${ }^{24}$

Furthermore, although air stacking is a therapy that can be taught quickly, with $>80 \%$ of participants accomplishing a stacked expired volume greater than FVC in this study, a training effect may exist. An observational study reported an increase in maximum insufflation capacity over 3-6-month intervals in $70 \%$ of people with a neuromuscular disease prescribed air stacking twice daily ${ }^{25}$; thus, familiarity with the therapy may account for some of the differences seen in cough peak flow achieved. Despite the broad range reported in the literature, the improvements seen in this present study align with the change from unassisted cough peak flow to air stacking-assisted cough peak flow values of $+35 \%$ to $+71 \%$ that other authors report. ${ }^{11,13,14,26} \mathrm{~A}$ recent study reported that air stacking can be learned by children with severe muscle weakness. ${ }^{27}$
In 18 subjects affected by spinal muscular atrophy and congenital muscular dystrophy, air stacking was shown to be an effective airway clearance therapy. However, air stacking was more effective in the absence of scoliosis.

To our knowledge, this is the first study to compare two different methods of insufflation used during air stacking. Our results suggest that both a ventilator and a resuscitator bag can be effective, improving the expired volume, cough peak flow, and $\mathrm{P}_{\text {Emax }}$ generated. Participants in the homeventilator group needed more successive insufflations to achieve maximum insufflation capacity compared with the resuscitator-bag group (2.6 vs 1.8 insufflations, respectively), probably due to the larger volume delivered with a bag compared with the set ventilator tidal volume. Although we did not measure leak with the resuscitator-bag method, mean ventilator leak was $<20 \%$ (289 $\mathrm{mL}$ ) of delivered volume.

Although the study protocol was designed to recruit participants who were naïve to air stacking to minimize bias, it is possible that some participants had discovered air stacking without being taught by professionals. By definition, participants were on ventilators that could perform air stacking, and some may also have possessed a bag for safety purposes. However, no participant had been prescribed air stacking or reported performing the therapy routinely or otherwise. Furthermore, the effect of performing daily air stacking on short-term outcomes, such as cough effectiveness, or longer-term lung function has not been studied in prospective randomized trials. It must be noted that this study did not compare air stacking and mechanical in-exsufflation. It is not known whether it would provide additional benefits beyond those seen here. Nonetheless, both techniques studied here are effective and produce cough peak flow in a clinically important range. 

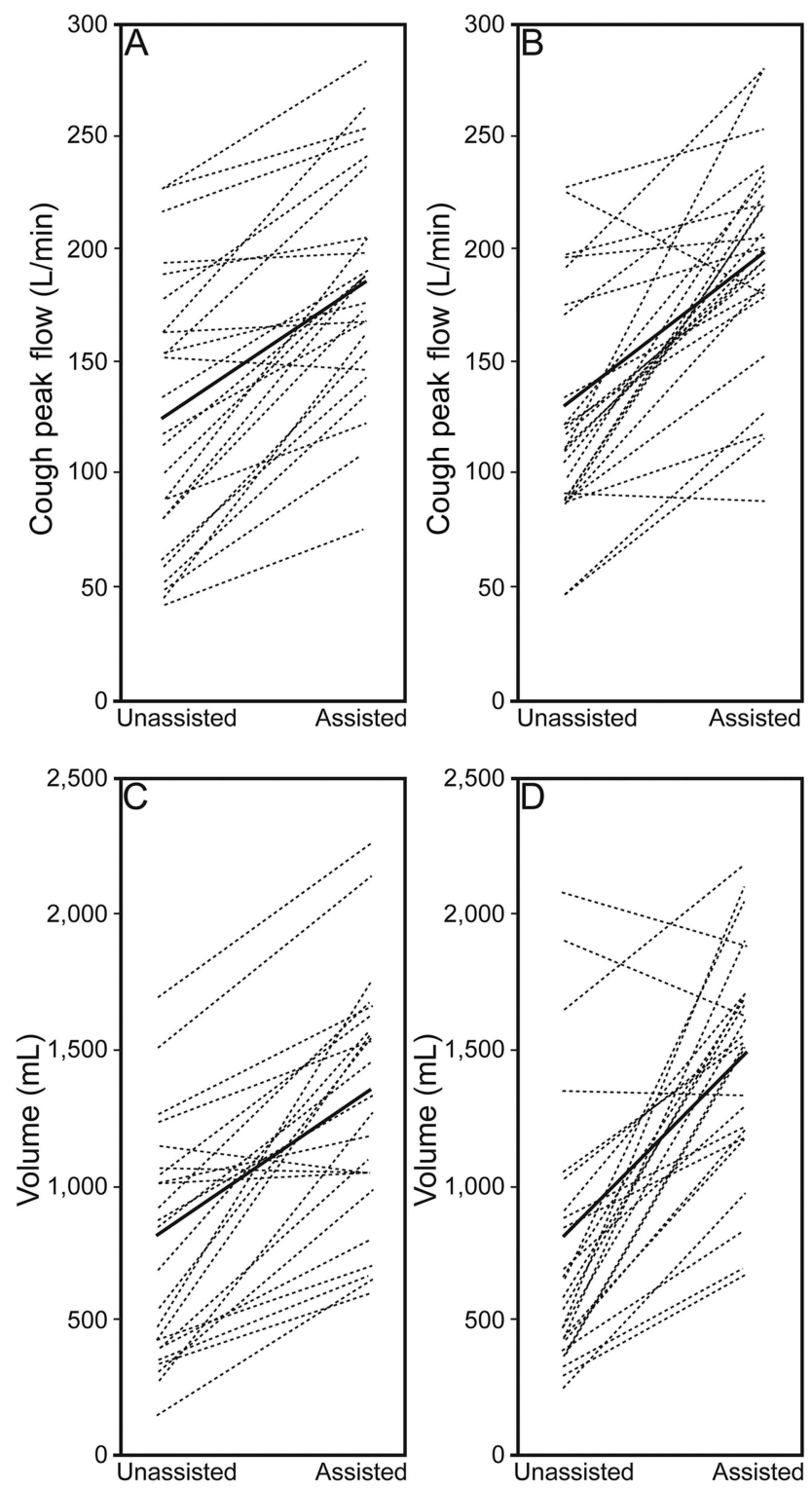

Fig. 2. Individual improvements in cough peak flow and volume with air stacking-assisted cough. A: Assisted cough peak flow with the resuscitator bag. B: Assisted cough peak flow with the ventilator. C: Assisted volume with the resuscitator bag. D: Assisted volume with the ventilator. The heavy dashed line represents the mean. 1: Unassisted values. 2: Assisted values. Heavy lines represent mean assisted values.

Cough augmentation is a crucial goal for people with neuromuscular disorders. A number of methods for improving cough effectiveness have been reported in the literature including manually assisted cough (ie, abdominal thrust), insufflation-assisted cough (using air-stacking techniques, positive pressure devices, or the insufflation component of a mechanical in-exsufflation device), exsufflation-assisted cough, or combinations of these. Although cough augmentation and air stacking techniques are recommended in many international clinical care guidelines, the frequency and timing of introduction and recommended techniques are highly variable, and no evidence is available. In this study, we found that air stacking-assisted cough peak flow produced similar values compared with participants' manually assisted cough peak flow, a finding that supports previous work in this area. ${ }^{10,22,23}$ Although we did not combine manually assisted coughing with air stacking in this study, prior data would suggest that combining these techniques to augment the different components of a cough has an even greater benefit on cough peak flow. ${ }^{10,22,23,28}$ However, the question of how to best achieve air stacking, either as a stand-alone technique or in combination, has not previously been addressed. Given the high cost of ventilators, positive pressure devices, and mechanical in-exsufflation machines, there is a need for a clinically comparable but cheaper alternative for cough augmentation. Our finding that air stacking via a resuscitator bag is as effective as air stacking via a ventilator suggests that an option does exist that may improve access to care for people with neuromuscular disorders.

\section{Conclusions}

Two techniques of cough augmentation via air stacking were investigated with participants randomized to receive air stacking either by a resuscitator bag or by a volumecycled ventilator. Short-term benefits of therapy were comparable, with similar air stacking-assisted cough peak flow values achieved irrespective of technique, suggesting that either a volume cycled ventilator or an inexpensive manual resuscitator bag can be used to improve cough effectiveness in people affected by DMD. Prospective randomized, controlled studies to investigate potential longer-term benefits of this therapy are recommended.

\section{REFERENCES}

1. Nigro G, Comi LI, Politano L, Bain RJ. The incidence and evolution of cardiomyopathy in Duchenne muscular dystrophy. Int J Cardiol 1990;26(3):271-277.

2. Konagaya M, Sakai M, Wakayama T, Kimura S, Kuru S, Yasuma F. Effect of intermittent positive pressure ventilation on life-span and causes of death in Duchenne muscular dystrophy. Rinsho Shinkeigaku 2005;45(9):643-646.

3. Rideau Y, Jankowski LW, Grellet J. Respiratory function in the muscular dystrophies. Muscle Nerve 1981;4(2):155-164.

4. Kohler M, Clarenbach CF, Bahler C, Brack T, Russi EW, Bloch KE. Disability and survival in Duchenne muscular dystrophy. J Neurol Neurosurg Psychiatry 2009;80(3):320-325.

5. Eagle M, Bourke J, Bullock R, Gibson M, Mehta J, Giddings D et al. Managing Duchenne muscular dystrophy: the additive effect of spinal surgery and home nocturnal ventilation in improving survival. Neuromuscul Disord 2007;17(6):470-475. 


\section{Cough Augmentation for Duchenne Muscular Dystrophy}

6. Bach JR, Ishikawa Y, Kim H. Prevention of pulmonary morbidity for patients with Duchenne muscular dystrophy. Chest 1997;112(4): 1024-1028.

7. Bach JR, Saporito LR. Criteria for extubation and tracheostomy tube removal for patients with ventilatory failure. A different approach to weaning. Chest 1996;110(6):1566-1571.

8. Chailleux E, Fauroux B, Binet F, Dautzenberg B, Polu JM. Predictors of survival in patients receiving domiciliary oxygen therapy or mechanical ventilation. A 10-year analysis of ANTADIR Observatory. Chest 1996;109(3):741-749.

9. Simonds AK. Respiratory support for the severely handicapped child with neuromuscular disease: ethics and practicality. Semin Respir Crit Care Med 2007;28(3):342-354

10. Toussaint M, Boitano LJ, Gathot V, Steens M, Soudon P. Limits of effective cough-augmentation techniques in patients with neuromuscular disease. Respir Care 2009;54(3):359-366.

11. Dohna-Schwake C, Ragette R, Teschler H, Voit T, Mellies U. IPPBassisted coughing in neuromuscular disorders. Pediatr Pulmonol 2006; 41(6):551-557.

12. Bach JR, Bianchi C, Vidigal-Lopes M, Turi S, Felisari G. Lung inflation by glossopharyngeal breathing and "air stacking" in Duchenne muscular dystrophy. Am J Phys Med Rehabil 2007;86(4):295300 .

13. McKim DA, Katz SL, Barrowman N, Ni A, LeBlanc C. Lung volume recruitment slows pulmonary function decline in Duchenne muscular dystrophy. Arch Phys Med Rehabil 2012;93(7):1117-1122.

14. Ishikawa Y, Bach JR, Komaroff E, Miura T, Jackson-Parekh R. Cough augmentation in Duchenne muscular dystrophy. Am J Phys Med Rehabil 2008;87(9):726-730.

15. Bach JR. Mechanical insufflation-exsufflation. Comparison of peak expiratory flows with manually assisted and unassisted coughing techniques. Chest 1993;104(5):1553-1562.

16. Jenkins HM, Stocki A, Kriellaars D, Pasterkamp H. Breath stacking in children with neuromuscular disorders. Pediatr Pulmonol 2014; 49(6):544-553.

17. Hull J, Aniapravan R, Chan E, Chatwin M, Forton J, Gallagher J et al. British Thoracic Society guideline for respiratory manage- ment of children with neuromuscular weakness. Thorax 2012; 67(Suppl 1):i1-i40.

18. Toussaint M, Soudon P, Kinnear W. Effect of non-invasive ventilation on respiratory muscle loading and endurance in patients with Duchenne muscular dystrophy. Thorax 2008;63(5):430-434.

19. Toussaint M, Steens M, Wasteels G, Soudon P. Diurnal ventilation via mouthpiece: survival in end-stage Duchenne patients. Eur Respir J 2006;28(3):549-555.

20. American Thoracic Society/European Respiratory Society. ATS/ERS Statement on respiratory muscle testing. Am J Respir Crit Care Med 2002;166(4):518-624.

21. Miller MR, Hankinson J, Brusasco V, Burgos F, Casaburi R, Coates A et al. Standardisation of spirometry. Eur Respir J 2005;26(2):319338

22. Kang SW, Kang YS, Moon JH, Yoo TW. Assisted cough and pulmonary compliance in patients with Duchenne muscular dystrophy. Yonsei Med J 2005;46(2):233-238.

23. Trebbia G, Lacombe M, Fermanian C, Falaize L, Lejaille M, Louis A et al. Cough determinants in patients with neuromuscular disease. Respir Physiol Neurobiol 2005;146(2):291-300.

24. Mellies U, Goebel C. Optimum insufflation capacity and peak cough flow in neuromuscular disorders. Ann Am Thorac Soc 2014;11(10): 1560-1568.

25. Kang SW, Bach JR. Maximum insufflation capacity. Chest 2000; 118(1):61-65

26. Brito MF, Moreira GA, Pradella-Hallinan M, Tufik S. Air stacking and chest compression increase peak cough flow in patients with Duchenne muscular dystrophy. J Bras Pneumol 2009;35(10):973979.

27. Marques TB, Neves Jde C, Portes LA, Salge JM, Zanoteli E, Reed UC. Air stacking: effects on pulmonary function in patients with spinal muscular atrophy and in patients with congenital muscular dystrophy. J Bras Pneumol 2014;40(5):528-534.

28. Torres-Castro R, Vilaró J, Vera-Uribe R, Monge G, Avilés P, Suranyi C. Use of air stacking and abdominal compression for cough assistance in people with complete tetraplegia. Spinal Cord 2014;52(5): 354-357. 